\title{
Interpretación fitosociológica de la vegetación de las lomas del desierto peruano
}

\author{
Antonio Galán de Mera ${ }^{1}$, Eliana Linares Perea ${ }^{2}$, José Campos de la $\mathrm{Cruz}^{3}$ \\ \& José Alfredo Vicente Orellana ${ }^{1}$ \\ 1. Departamento de Biología (Botánica), Universidad San Pablo-CEU, apartado 67, 28660- Boadilla del Monte, Madrid, \\ España; agalmer@ceu.es, avicore@ceu.es \\ 2. Estudios Fitogeográficos del Perú, Sánchez Cerro 219, Manuel Prado, Paucarpata, Arequipa, Perú; \\ elialinper@hotmail.com \\ 3. Museo de Historia Natural, Universidad Nacional Mayor de San Marcos, apartado 140434, Lima-14, Perú; \\ joricampos@yahoo.es
}

Recibido 04-VI-2010. Corregido 01-XI-2010. Aceptado 07-XII-2010.

\begin{abstract}
A phytosociological interpretation of vegetation from sandy hills of the Peruvian desert. The vegetation of the sandy hills ("lomas") constitutes the main originality of the Peruvian and Chilean desert with a high number of endemics that shapes the vicarious associations. In this work, a phytosociological view of sandy environments of the Peruvian coastal desert is presented. According to the Braun-Blanquet method, we have made up 32 phytosociological inventories and added 138 ones from others authors. In each inventory, we have analyzed its floristic composition and ecological parameters, as altitude, soil and geomorphology. All releves were synthesized in a table to deduce the different associations, higher phytosociological units, and the distribution of its flora along the Peruvian coast and the Andean Cordillera. Using the Shannon-Wiener diversity index, the diversity of this flora is discussed making a comparison with historical data about the use of the territory with livestock during pre-Inca and Inca cultures, and Spanish invasion. As a result, two associations from Southern Peru-Nolanetum scaposo-spathulatae and Palauetum camanensis-weberbaueri-, two alliances -Nolanion humifusae from central Peru, and Nolanion spathulatae from the Southern Peru- and a new order -Tetragonio crystallinae-Plantaginetalia limensis- are described. In Nolanetum scaposo-spathulatae, Dictyophragnus englerianus, Leptoglossis lomana, Nolana scaposa, N. spathulata, Palaua velutina and Tetragonia vestita are the main characteristics, while in Palauetum camanensis-weberbaueri association N. scaposa and P. velutina are replaced by Palaua camanensis and P. weberbaueri. Nolanion humifusae alliance integrates species as Geranium limae, Hymenocallis amancaes, Nolana humifusa, N. latipes, Palaua rhombifolia or Villanova oppositifolia. Likewise, Cistanthe weberbaueri, Cryptantha parviflora, Hoffmannseggia miranda, Lupinus mollendoensis, Nolana confinis, N. pallidula, N. scaposa, N. spathulata, Palaua camanensis, P. velutina, P. weberbaueri, Tetragonia vestita and Weberbauerella brongniartioides are the characteristic species of Nolanion spathulatae alliance. The Tetragonio crystallinae-Plantaginetalia limensis order presents characteristic plants don't linked with eutrophic soils, as Calandrinia alba, Cryptantha limensis, Dyschoriste repens, Monnina macrostachya, Oxalis lomana, Palaua malvifolia, Pectocarya lateriflora, Plantago limensis or Tetragonia crystallina, with a distribution that claps the geographical area of the new alliances. On the other hand, the vegetation of the desert ravines is discussed in the context of the coastal river plant communities and its disturbance by the dunes. After the application of the Shannon-Wiener diversity index on the synthetic table columns, we can deduce that an increase in Andean and European ruderal species is linked to an intensive livestock activity. The transhumance between the Andes and the coast from pre-Inca times until now, produces the plant dispersion of high Andean plants toward the coast; the Spanish colonization was the origin of the presence of European plants in the "lomas" vegetation of Peru. Rev. Biol. Trop. 59 (2): 809-828. Epub 2011 June 01.
\end{abstract}

Key words: Peru, desert, phytosociology, diversity, pre-Inca/Inca cultures, Spanish invasion, use of the territory. 
La formación de las lomas de la costa peruana y chilena $\left(29^{\circ} 55^{\prime} \mathrm{W}-6^{\circ} \mathrm{S}\right)$ constituye la mayor originalidad del Desierto Pacífico (Rundel et al. 1991) puesto que, se debe a dos situaciones particulares de esas latitudes: primero las nieblas causadas por la corriente fría de Humboldt, entre junio y octubre, segundo, el aumento de las lluvias esporádicas causadas por el fenómeno de El Niño a lo largo del desierto peruano y chileno (Weberbauer 1912, 1936, Ferreyra 1960, 1993, Arntz \& Fahrbach 1996, Brack Egg \& Mendiola Vargas 2004). Entonces, las nieblas precipitan en algunos puntos entre 250 y $1000 \mathrm{~m}$ de altitud y originan los diferentes tipos de vegetación de las lomas, que en realidad se desarrollan en áreas aisladas "Nebeloasen": oasis de nieblas de Ellenberg (1959)- a lo largo del Desierto Pacífico que propician la presencia de un buen número de endemismos y forman asociaciones vicariantes (Müller 1985, Galán de Mera et al. 1997). Así, la mayor riqueza endémica la alcanza el género Nolana L.f. (Nolaneae, Solanaceae) con 92 especies (Dillon et al. 2007), y la mayor cantidad de endemismos pertenecientes a distintos géneros se concentra entre los departamentos de Arequipa, Moquegua y Tacna (Galán de Mera et al. 1997). Una cartografía de estas islas de vegetación en el Desierto Pacífico podemos encontrarla en el mapa de Hueck \& Seibert (1981).

El levantamiento de inventarios fitosociológicos para comparar la vegetación de distintos territorios se va haciendo cada vez más frecuente en América Central, El Caribe y América del Sur (Huber \& Riina 1997, Galán de Mera 2005), incluso en los bosques amazónicos (Phillips \& Miller 2002), y que van orientados a conocer la biodiversidad de las regiones.

Aunque existen numerosos trabajos sobre la flora de las lomas del Perú desde que Tafalla iniciara su estudio en las lomas de Atiquipa (Arequipa) en el siglo XVIII (Weberbauer 1912), fue Weberbauer (1945) quien comenzó las observaciones de la vegetación y se basó en la flora y en parámetros climáticos, y que posteriormente fueron sintetizadas por otros autores (Müller \& Müller 1974, Müller 1985, Galán de Mera et al. 2002, 2009) con el uso del método fitosociológico de Braun-Blanquet (1932). Así, Müller \& Müller (1974) realizan una primera aproximación a las formaciones vegetales de la costa peruana, profundizada posteriormente por Müller (1985) al describir 14 nuevas asociaciones en el departamento de Lima que en la mayoría de los casos, no incluye en unidades fitosociológicas superiores precisas. Galán de Mera et al. (2002) crean, por tanto, la asociación Philoglosso peruvianaeUrocarpidetum peruviani, la alianza Loasion urentis y el orden Commelinetalia fasciculatae que reúnen a la vegetación nitrófila y subnitrófila andina y pacífica dentro de la clase Stellarietea mediae $\mathrm{R}$. Tx., Lohmeyer \& Preising ex von Rochow 1951. Puesto que ésta es extratropical, Galán de Mera (2005) describe la clase Palauo dissectae-Nolanetea gayanae para la vegetación anual de las lomas del Desierto Pacífico, que además queda enriquecida con la descripción de las asociaciones Hoffmannseggio mirandae-Palauetum weberbaueri y Nolano spathulatae-Palauetum dissectae (Galán de Mera et al. 2009).

Los trabajos que pretenden relacionar la estructura y composición florística de las comunidades vegetales con el uso que ha dado el hombre a los territorios tanto en Europa (Jones 1988, Wilmanns 1997, Galán de Mera et al. 2000) como en África (Deil 2003), o América (Beresford-Jones et al. 2009, Young 2009) constituyen diferentes aportes a la etnogeobotánica. En el caso de las culturas preincaicas e Inca, basaban su economía en el control vertical de un máximo de pisos ecológicos entre la costa y las alturas de los Andes (Murra 2009), donde por supuesto era muy importante la trashumancia (Díez de San Miguel 1964) lo que sin duda, hace interesante su relación con la variabilidad de las asociaciones y el origen de su flora. La trashumancia se lleva a cabo en la actualidad, pero la idea del espacio de la colonización española fue muy diferente a la distribución altitudinal de la producción preincaica e incaica. Los españoles hablan horizontalmente de "costa, sierra y selva" (Cieza de 
León 1988), y en cada región deben estar dispuestas las poblaciones y sus actividades, por lo que nacen las reducciones y rompen la continuidad de uso de los pisos ecológicos (Sluyter 2001). Las lomas del desierto quedaron bajo el régimen de los latifundios creados a lo largo de la costa peruana y chilena, como un reservorio de pastos para los distintos tipos de ganadería aportados durante la colonia (Puig et al. 2002).

El objetivo del presente trabajo es dar a conocer nuevas asociaciones de la vegetación efímera de las lomas del Perú y, como resultado de la comparación florística con los resultados de otros autores, determinar cómo se agrupan en alianzas y sus unidades fitosociológicas superiores. Una segunda finalidad es observar cómo contribuyen las diferentes especies a la diversidad de las comunidades de plantas relacionándolas con el uso ganadero de las lomas.

\section{MATERIAL Y MÉTODOS}

En el presente trabajo se han estudiado las lomas situadas entre el sur (S) de Lima (18L 0273922, 8675599), la punta de Lachay (18L $0224230,8765461)$ y algunas localidades situadas en Ica (Cerros de Quilmaná- 18L 0351723, 8568458) y Trujillo (Cerro Campana- 17M 0708317, 9119187). En ellas son frecuentes los depósitos eólicos y los materiales cretácicos sedimentarios (IGM 1975). En el S del Perú, hemos levantado inventarios en las lomas de Mejía (19K 0192035, 8107927) y Camaná (18K 0746617, 8165622), donde los aportes eólicos se han depositado sobre materiales metamórficos y sedimentarios del Terciario. El desierto en el S tiene como característica geomorfológica la presencia de una gran llanura muy árida, con sedimentación fluvial, que se extiende entre 1000-2000m de altitud, y con el aporte de otros trabajos, lo hemos considerado como una gran barrera biogeográfica interdesértica (Galán de Mera et al. 1997, 2009).

De acuerdo con la aproximación bioclimática de Rivas-Martínez et al. (1999), la región de estudio se encuentra en el piso bioclimático termotropical (Índice de termicidad 490-730) hiperárido (5-30mm). Sin embargo, hay lugares de la costa peruana donde por precipitación de las nieblas causadas por la corriente de Humboldt, podemos alcanzar un ombroclima árido (30-100mm) (Atico- 18K 0659814, 8201232, Cachendo- 19K 0204895, 8118654) con la presencia de comunidades de cactáceas columnares con Neoraimondia arequipensis Backeb., e incluso seco (300-500mm) en Lachay (Lima) o Atiquipa (18L 0568805, 8260265) en Arequipa, donde podemos observar bosques de Caesalpinia spinosa (Molina) Kuntze y Myrcianthes ferreyrae (McVaugh) McVaugh respectivamente.

En el presente trabajo, hemos utilizado el método de Braun-Blanquet (1932), que se basa en el establecimiento de asociaciones de inventarios con una estimación de la abundancia de elementos florísticos en un biotopo o espacio geomorfológico determinado (Schwabe 1997) medido en metros cuadrados. El estudio de asociaciones donde se han introducido plantas ligadas al uso del territorio por el hombre o los animales llevó a la interpretación de la variabilidad de las asociaciones con los conceptos de subasociación, comunidad basal y comunidad derivada (Schaminée \& Stortelder 1996), y comunidad marginal (Foucault 1981, Dierschke 1994, Kopecký et al. 1995). La subasociación se debe a pequeñas diferencias locales de la asociación, como un aumento de la inclinación o una mayor pedregosidad; la comunidad basal está formada por las plantas pioneras de la asociación, que suelen pertenecer a las unidades fitosociológicas superiores a la asociación; la comunidad derivada supone la introducción de plantas ligadas a la acción antrópica con la consiguiente pérdida de especies de la asociación; y las comunidades marginales reflejan la pérdida de plantas de la asociación o de sus unidades superiores al alejarnos del areal geográfico o de las condiciones ecológicas propias de la asociación.

Los 32 inventarios fitosociológicos con los que podemos conocer las diferentes comunidades efímeras de las lomas han sido obtenidos directamente en el campo, a los que sumamos los 138 obtenidos por otros autores (Müller 1985, Galán de Mera et al. 2002) a lo largo 
del desierto costero peruano. Con el programa SORT 4.1 (Ackermann \& Durka 1997) hemos ordenado los inventarios de acuerdo con la frecuencia y distribución de las plantas presentes. El resultado es un análisis entre los datos de campo y lo publicado por otros autores, cuyos inventarios disponemos en una tabla sintética
(Cuadro 1). Las columnas del Cuadro 1 proceden, a su vez, de sintetizar tablas de inventarios según la constancia que tienen las plantas en cada uno de ellos.

Con el programa SORT 4.1 se puede calcular el índice de diversidad de Shannon-Wiener, especialmente útil cuando se han tomado más

\section{CUADRO 1}

Inventarios propios y de otros artículos

TABLE 1

Releves of the authors and from other papers

Número de comunidad

Inventarios por comunidad

Diversidad (Shannon-Wiener)

Características de asociaciones y alianzas

Villanova oppositifolia

Nolana humifusa

Erigeron leptorhizon

Oxalis bulbigera

Palaua rhombifolia

Hymenocallis amancaes

Drymaria weberbaueri

Galinsoga caligensis

Geranium limae

Haageocereus olowinskianus

Centaurium lomae

Nolana latipes

Loxanthocereus gracilispinus

Nolana spathulata

Leptoglossis lomana

Palaua weberbaueri

Nolana confinis

Palaua velutina

Spergularia congestifolia

Dictyophragmus englerianus

Cryptantha parviflora

Tiquilia elongata

Cistanthe weberbaueri

Hoffmannseggia miranda

Weberbauerella brongniartioides

Haageocereus decumbens
111111111122222222

123456789012345678901234567

1

1

1

852077425642255324854325332

212222011222222333232212222

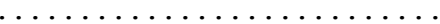

291237637980328020919272536

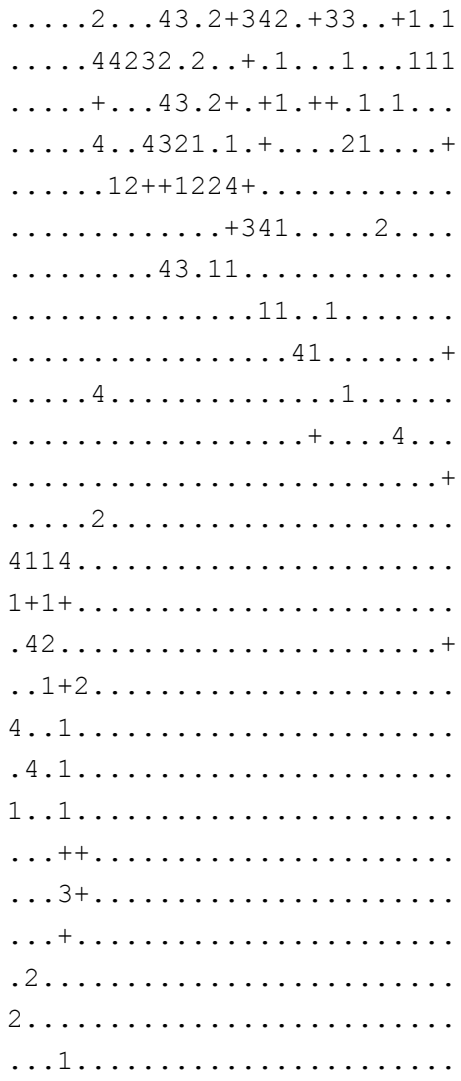


CUADRO 1 (Continuación)

Inventarios propios y de otros artículos

TABLE 1 (Continued)

Releves of the authors and from other papers

Número de comunidad

Nolana pallidula

Lupinus mollendoensis

Nolana scaposa

Palaua camanensis

Tetragonia vestita

Oxalis sepalosa

Malesherbia angustisecta

Características de Loasion y Commelinetalia

Loasa urens

Philoglossa peruviana

Loasa nitida

Exodeconus prostratus

Características de orden y clase

Eragrostis peruviana

Plantago limensis

Oenothera arequipensis

Cryptantha granulosa

Rostraria trachyantha

Pectocarya lateriflora

Nolana gayana

Tetragonia crystallina

Calandrinia alba

Oxalis megalorrhiza

Palaua dissecta

Cryptantha limensis

Dyschoriste repens

Spananthe peruviana

Cristaria multifida

Linum parvum

Palaua malvifolia

Vicia lomensis

Oxalis solarensis

Monnina macrostachya

Argylia radiata

Tiquilia litoralis

Verbena pogostoma

Cistanthe celosioides

Gilia glutinosa

Loasa fruticosa

Tiquilia dichotoma

Oxalis lomana
111111111122222222

123456789012345678901234567

$1 \ldots \ldots \ldots \ldots \ldots$

$2 \ldots \ldots \ldots \ldots \ldots$

$\ldots 2 \ldots \ldots \ldots \ldots \ldots$

$.2 \ldots \ldots \ldots \ldots \ldots$

$\ldots 2 \ldots \ldots \ldots \ldots \ldots$

$\ldots 1 \ldots \ldots \ldots \ldots \ldots$

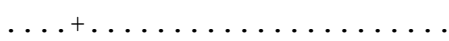

$.+1+\ldots .+\ldots+\ldots \ldots \ldots . .212$

$\ldots \ldots \ldots+2433 \ldots \ldots$

$\ldots \ldots \ldots \ldots 232 \ldots$

$\ldots \ldots \ldots \ldots \ldots \ldots \ldots \ldots$

$1411 \ldots .44 .224+.1 \ldots \ldots 121$

$211+\ldots 1.3312+\ldots \ldots \ldots \ldots 1$

$++1+\ldots 2.33222 \ldots \ldots \ldots \ldots$

$\ldots \ldots+\ldots . .2 .12231 \ldots 32 \ldots \ldots+$

$\ldots \ldots+\ldots+\ldots+432 \ldots 3 \ldots+1 .+$

$\ldots \ldots \ldots \ldots 1 \ldots 2221141 \ldots \ldots 1$

$\ldots+\ldots 1.34121+\ldots \ldots \ldots \ldots 1$

$\ldots 2+\ldots 1+\ldots 1 \ldots \ldots \ldots \ldots 2$

$\ldots \ldots \ldots 2 \ldots+.+.1 \ldots 1 \ldots 1$

$\ldots \ldots \ldots+\ldots \ldots \ldots 1 \ldots 43 .+\ldots$

$41.4 \ldots \ldots \ldots \ldots \ldots \ldots$

$\ldots \ldots \ldots 1 \ldots 1 \ldots \ldots 1 \ldots+$

$\ldots \ldots \ldots 1332 \ldots \ldots$

$\ldots \ldots \ldots \ldots 241 \ldots \ldots$

$\ldots 1 \ldots \ldots 2 .+\ldots \ldots \ldots$

$\ldots \ldots \ldots+\ldots+3 \ldots \ldots \ldots$

$\ldots \ldots \ldots .+\ldots \ldots \ldots+$

$\ldots \ldots \ldots \ldots \ldots+\ldots \ldots \ldots$

$\ldots \ldots 2 \ldots \ldots \ldots \ldots$

$3 \ldots \ldots \ldots \ldots \ldots \ldots$

$\ldots 1 \ldots \ldots \ldots \ldots \ldots$

$\ldots \ldots \ldots \ldots \ldots \ldots$

$\ldots+\ldots \ldots \ldots \ldots \ldots \ldots$

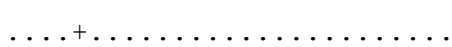

$\ldots+\ldots \ldots \ldots \ldots \ldots \ldots$

$\ldots \ldots+\ldots \ldots \ldots \ldots \ldots \ldots$

$\ldots+\ldots \ldots \ldots \ldots \ldots \ldots$

$\ldots \ldots \ldots \ldots \ldots \ldots+$ 
CUADRO 1 (Continuación)

Inventarios propios y de otros artículos

TABLE 1 (Continued)

Releves of the authors and from other papers

Número de comunidad

\section{Comunidades de quebradas}

Atriplex rotundifolia

Ephedra americana

Lycopersicon pennellii

Ambrosia artemisioides

Schinus molle

Tessaria absinthioides

Chamaesyce serpens

Ophryosporus hoppii

Alternanthera arequipensis

Tarasa operculata

Especies ruderales autóctonas

Parietaria debilis

Cyclospermum laciniatum

Urocarpidium peruvianum

Chenopodium petiolare

Solanum montanum

Nicotiana paniculata

Salvia rhombifolia

Valeriana chaerophylloides

Acmella alba

Paspalum penicillatum

Urocarpidium leptocalyx

Commelina fasciculata

Daucus montanus

Eragrostis mexicana

Solanum phyllanthum

Lycopersicon peruvianum

Ciclospermum leptophyllum

Galinsoga parviflora

Acmella oleracea

Solanum multifidum

Solanum americanum

Sida jatrophioides

Valeriana interrupta

Bowlesia palmata

Commelina hispida

Urocarpidium chilense

Ipomoea tricolor

\section{Especies introducidas}

Sonchus oleraceus

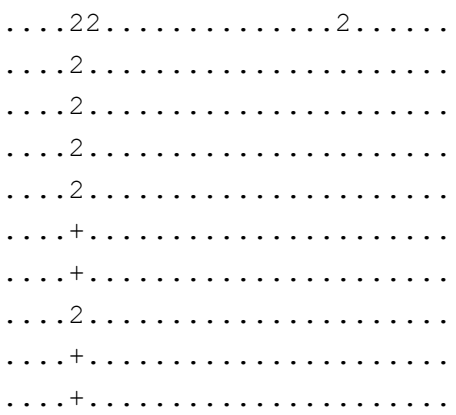

....3..43.11232122322.232

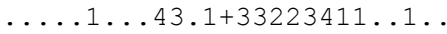

$\ldots \ldots \ldots 22 \ldots 4442 .+31 \ldots 334$

$.122 .+\ldots \ldots 1+1 \ldots \ldots \ldots 311$

$\ldots .4 \ldots 442 \ldots+321 \ldots 2$

$\ldots \ldots 3 \ldots \ldots 1.11 \ldots 22 \ldots 334$

$\ldots \ldots \ldots 24.2111 \ldots 1 \ldots . \ldots 1$

$\ldots \ldots \ldots \ldots++2444 \ldots \ldots+$

$\ldots \ldots \ldots 21 \ldots+.1 \ldots+21 \ldots \ldots$

$\ldots \ldots \ldots \ldots+12334 \ldots+\ldots$

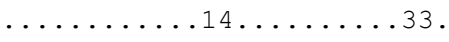

$\ldots \ldots \ldots \ldots \ldots \ldots 21 \ldots 2$

$\ldots \ldots \ldots \ldots 242 \ldots \ldots$

$\ldots \ldots \ldots 211 \ldots \ldots \ldots$

$\ldots \ldots \ldots \ldots \ldots \ldots \ldots \ldots$.

$\ldots \ldots \ldots \ldots \ldots+\ldots \ldots 1$

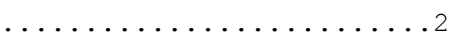

$\ldots \ldots \ldots \ldots \ldots \ldots+$
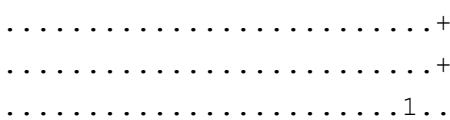

$\ldots \ldots \ldots \ldots \ldots \ldots \ldots$
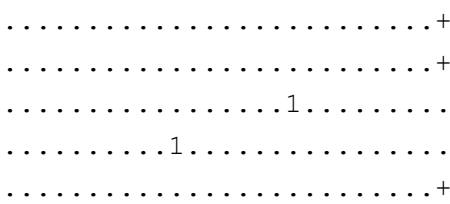

$+.1 \ldots+\ldots+12 \ldots 12424442$.322+ 
CUADRO 1 (Continuación)

Inventarios propios y de otros artículos

TABLE 1 (Continued)

Releves of the authors and from other papers

\section{Número de comunidad}

Erodium moschatum

Erodium malacoides

Medicago polymorpha

Erodium cicutarium

Chenopodium murale

Poa аппиа

Anagallis arvensis

Euphorbia peplus

Emex spinosa

Stachys arvensis

Veronica persica

Sisymbrium irio

Cotula australis

Polycarpon tetraphyllum

Lophochloa cristata

Torilis nodosa

Silene gallica

Lamarckia aurea

Centunculus minimus

Gamochaeta purpurea

Fumaria agraria

Cerastium glomeratum

Urtica urens

Linaria canadensis

Briza minor

Dichondra cf. donelliana

Fumaria parviflora

Sagina apetala

Legousia biflora

Capsella bursa-pastoris

Fumaria capreolata

Elementos fisurícolas

Adiantum subvolubile

Begonia octopetala

\section{Compañeras}

Talinum paniculatum

Stenomesson croceum

Croton alnifolius

Anthericum eccremorrhizum

Oxalis latifolia

Trixis paradoxa
111111111122222222

123456789012345678901234567

......22..+3421141.1.1.

$\ldots \ldots \ldots \ldots+121+3 \ldots \ldots 11$.

$\ldots \ldots \ldots+\ldots 222343 \ldots 1 \ldots$

$\ldots \ldots 1 \ldots 32 \ldots 323 \ldots 1 \ldots$

$\ldots \ldots \ldots 1 \ldots+1 \ldots 11 \ldots 33$.

.........1133...1.+

.........2342...2.+

$\ldots \ldots \ldots \ldots+212+\ldots+\ldots$

$\ldots \ldots \ldots 1 \ldots 4+1 \ldots \ldots 2 \ldots$

$\ldots \ldots \ldots \ldots \ldots+\ldots \ldots 1+$

$\ldots \ldots \ldots \ldots 443 \ldots+\ldots$

$\ldots \ldots \ldots \ldots+\ldots \ldots 22$.

$\ldots \ldots \ldots \ldots \ldots \ldots+\ldots 1$

$\ldots \ldots \ldots \ldots \ldots 11 \ldots 4 \ldots$

$\ldots \ldots \ldots \ldots \ldots$

$\ldots \ldots \ldots \ldots \ldots$

$\ldots \ldots \ldots \ldots 11 \ldots+\ldots \ldots$

$\ldots \ldots \ldots 11 \ldots \ldots \ldots 1 \ldots$

$\ldots \ldots \ldots \ldots \ldots \ldots$

$.+\ldots \ldots \ldots \ldots \ldots \ldots+$

$\ldots \ldots \ldots \ldots+1 \ldots \ldots \ldots$

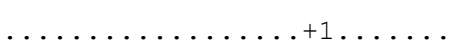

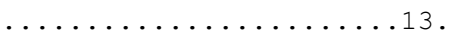

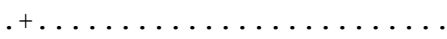

$\ldots \ldots \ldots \ldots \ldots \ldots$

$\ldots \ldots \ldots \ldots \ldots+\ldots \ldots$

$\ldots \ldots \ldots \ldots \ldots \ldots \ldots$

$\ldots \ldots \ldots \ldots \ldots \ldots \ldots$

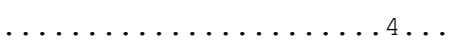

$\ldots \ldots \ldots \ldots \ldots \ldots \ldots$

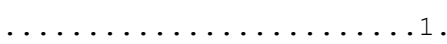

$\ldots \ldots \ldots \ldots+1411 \ldots \ldots$

$\ldots \ldots \ldots \ldots \ldots \ldots$

$1+.1 .2 \ldots 42111 \ldots \ldots \ldots \ldots+$

$\ldots .4 \ldots 1 .+++12++2 \ldots \ldots$

$\ldots \ldots \ldots++32434 \ldots 1 \ldots$

$\ldots .4 \ldots++\ldots 1 \ldots 2 \ldots \ldots \ldots+$

$\ldots \ldots \ldots 1211 \ldots \ldots+$

$\ldots \ldots \ldots 321 \ldots \ldots 2 \ldots+$ 
CUADRO 1 (Continuación)

Inventarios propios y de otros artículos

TABLE 1 (Continued)

Releves of the authors and from other papers

Número de comunidad
Vulpia megalura
Camassia biflora
Cuscuta sp.
Solanum sp.
Calceolaria pinnata
Sicyos baderoa
Heliotropium arborescens
Eragrostis nigricans
Hoffmannseggia prostrata
Senecio abadianus
Ophryosporus pubescens
Euphorbia elliptica
Begonia geraniifolia
Sigesbeckia flosculosa
Onoseris odorata
Gnaphalium sp.

111111111122222222

123456789012345678901234567

$\ldots \ldots+\ldots \ldots \ldots \ldots 11+\ldots+\ldots$

$\ldots .2 \ldots 3 \ldots+.+\ldots 4 \ldots \ldots$

$\ldots 12.1 \ldots \ldots \ldots 1 \ldots \ldots$

$\ldots \ldots \ldots 311.2 \ldots \ldots \ldots$

...............

$\ldots \ldots \ldots \ldots \ldots 1 \ldots 1 \ldots 11$

$\ldots \ldots \ldots 2414 \ldots \ldots$

$2423 \ldots \ldots \ldots \ldots \ldots \ldots$

$4+.11 \ldots \ldots \ldots \ldots \ldots \ldots$

$\ldots \ldots+\ldots \ldots \ldots 11 \ldots+\ldots \ldots$

$\ldots \ldots \ldots \ldots 31 .+\ldots 1 \ldots$

$\ldots \ldots \ldots \ldots \ldots+\ldots 22 \ldots \ldots$

$\ldots \ldots \ldots \ldots+11 \ldots \ldots \ldots$

$\ldots \ldots \ldots \ldots 21 \ldots+\ldots \ldots$

$1.12 \ldots \ldots \ldots \ldots \ldots$

$\ldots \ldots \ldots 1 \ldots \ldots+\ldots 1 \ldots \ldots$

Compañeras con una o dos presencias: Encelia canescens 1:1, 15:1; Galinsoga sp. 3:1, 4:1; Alstroemeria pelegrina 6:+, 21:3; Boussingaultia diffusa 6:2, 21:2; Cladonia aff. rangiformis 9:3, 10:3; Euphorbia sp. 10:1, 11:2;Oenothera nocturna 16:+, 17:1; Heliotropium angiospermum 16:+, 17:1; Acnistus arborescens 18:4, 19:+; Nostoc commune 19:+, 24:2; Musci div. spec. 19:1, 24:4; Dicliptera tomentosa 19:+, 20:+; Hyptis sidifolia 19:+, 21:1; Stipa mucronata 19:1, 24:+; Andrachne microphylla 21:2, 22:2; Portulaca perennis 1:+; Cenchrus brownii 1:4; Nothoscordum bivalve 2:1; Grindelia glutinosa 2:4; Heliotropium krauseanum 4:+; Peperomia hillii 6:+; Cladonia sp. 6:4; Tillandsia latifolia 6:+; Crassula connata 10:+; Cyclanthera mathewsii 11:1; Calandrinia sp. 15:+; Encelia sp. 15:+; Oxalis micrantha 16:+; Lantana scabiosiflora 17:1; Tournefortia microcalyx 17:1; Verbena litoralis 18:1; Cyperus hermaphroditus 18:1; Carica candicans 18:2; Jaltomata umbellata 19:+; Hepaticae div. spec. 19:+; Salvia tubiflora 21:1; Browallia grandiflora 21:1; Ipomoea dubia 21:2; Tropaeolum minus 27:+; Dioscorea chancayensis 27:+; Stenomesson coccineum 27:+; Spananthe paniculata 27:+; Suaeda foliosa 27:+; Drymaria paposana 27:1; Stellaria cuspidata 27:1; Luffa operculata 27:+; Heliotropium lanceolatum 27:1.

Índices de constancia: +: < 10\%, 1: 11 a 20\%, 2: 21 a 40\%, 3: 41 a 60\%, 4: 61 a 80\%, 5: > 81\%.

de diez réplicas en una tabla, para observar las variaciones que existen entre cada comunidad, las cuales coinciden con cada una de las columnas (Magurran 1989).

Para la interpretación de la distribución de la flora hemos considerado las aportaciones de numerosos autores, como Bruns (1929), Weberbauer (1945), Ferreyra (1953, 1960, 1983), Müller \& Müller (1974), Müller (1985), Rundel et al. (1991), Galán de Mera et al. (1997), Puig et al. (2002), Tutin et al.
(1964-1980), Flora of North America Committee (1992-2007).

La nomenclatura y autorías de los taxones tratados están de acuerdo con Brako \& Zarucchi (1993), actualizados en Solomon (2010). Los sintáxones atienden al Código Internacional de Nomenclatura Fitosociológica (Izco \& Del Arco 2003). Ahora bien, para la determinación de las plantas se han consultado los herbarios AQP, F, MO, NY, US, USM y USP; los especímenes recolectados se conservan en AQP. 


\section{RESULTADOS}

1. Asociaciones y unidades de vegetación. Aspectos sintaxonómicos: El Cuadro 1 recoge una síntesis de todas las comunidades vegetales que han sido inventariadas en las localidades del Cuadro 2; en el Cuadro 1, además, hemos distinguido las características de asociación y alianza de acuerdo con su distribución en el S del Perú (entre los departamentos de Ica y Tacna) o en el centro (desde La Libertad a Lima). Naturalmente, las especies características de orden y clase presentan una distribución muy amplia, adentrándose incluso en las lomas chilenas, como ocurre por ejemplo con Cristaria multifida Cav., Loasa urens Jacq. o Rostraria trachyantha (Phil.) Tzvelev ex Soreng.

Es de particular importancia la distribución del género Nolana (Dillon et al. 2007): $N$. humifusa (Gouan) I.M. Johnst., desde Trujillo a Lima, y N. spathulata Ruiz \& Pav., desde Arequipa a Tacna. N. humifusa y N. spathulata definen dos nuevas alianzas fitosociológicas junto con las plantas que las acompañan.

\subsection{Nolanion humifusae all. nova (holo-} typus: Tetragonio crystallinae-Nolanetum gayanae Müller 1985): Reúne a las comunidades anuales no nitrófilas de las lomas que se extienden entre Trujillo y Lima. Son sus especies características: Centaurium lomae (Gilg) Druce, Drymaria weberbaueri Muschl., Erigeron leptorhizon DC., Galinsoga caligensis Canne, Geranium limae R. Knuth, Hymenocallis amancaes (Ruiz \& Pav.) G. Nicholson, Oxalis bulbigera R. Knuth, Nolana humifusa, $N$. latipes I.M. Johnst., Palaua rhombifolia R. Graham y Villanova oppositifolia Lag..

Por consiguiente, Müller (1985) indica que en las lomas situadas en los alrededores de Lima existen dos asociaciones que alcanzan una mayor extensión, Tetragonio crystallinae-Nolanetum gayanae, de suelos arenosos poco estabilizados (Cuadro 1, columnas 6-11) y Palauo rhombifoliae-Nolanetum gayanae, de suelos arenosos consolidados (Cuadro 1, columnas 12-20).
1.2. Nolanion spathulatae all. nova (holotypus: Nolano spathulatae-Palauetum dissectae Galán de Mera, Linares, Campos \& Vicente Orellana 2009): Reúne a las comunidades efímeras no nitrófilas de las lomas que se extienden entre Ica y Tacna. Son sus especies características: Cistanthe weberbaueri (Diels) Carolin ex Hershkovitz, Cryptantha parviflora Reiche, Dictyophragmus englerianus (Muschl.) O.E. Schulz, Hoffmannseggia miranda Sandwith, Leptoglossis lomana (Diels) Hunz., Lupinus mollendoensis Ulbr., Nolana confinis (I.M. Johnst.) I.M. Johnst., N. pallidula I.M. Johnst., N. scaposa Ferreyra, N. spathulata, Palaua camanensis Ferreyra \& Chanco, $P$. velutina Ulbr. \& Hill, P. weberbaueri Ulbr., Spergularia congestifolia I.M. Johnst., Tetragonia vestita I.M. Johnst., Tiquilia elongata (Rusby) A.T. Richardson y Weberbauerella brongniartioides Ulbr.

Debido a la mayor diversidad de endemismos en el desierto del sur del Perú (Galán de Mera et al. 1997, Dillon et al. 2007) podemos reconocer un número mayor de asociaciones entre las lomas de Mejía y de Camaná.

\subsubsection{Nolano spathulatae-Palauetum} dissectae Galán de Mera, Linares, Campos \& Vicente Orellana 2009 (Cuadro 1, columna 1; Cuadro 3, inventarios 1-8): Esta vegetación terofítica tapiza las lomas de Mejía (Fig. 1A), y se desarrolla hasta $200 \mathrm{~m}$ de altitud sobre suelos arenosos poco estabilizados, los cuales son resultantes de aportes eólicos (Galán de Mera et al. 2009). Destacamos en su combinación florística a Lupinus mollendoensis, Nolana pallidula, N. spathulata y Weberbauerella brongniartioides.

\subsubsection{Hoffmannseggio mirandae-Palaue-} tum weberbaueri Galán de Mera, Linares, Campos \& Vicente Orellana 2009 (Cuadro 1, columna 2; Cuadro 3, inventarios 9-13): Se diferencia de la anterior porque se instala sobre arenas consolidadas a mayor altitud $(300-400 \mathrm{~m})$, ocupando las zonas interiores de las lomas de Camaná (Fig. 1B). Son especies características: 


\section{CUADRO 2}

Comunidades y procedencia de las columnas del cuadro 1

TABLE 2

Plant communities and references of the columns of table 1

\begin{tabular}{|c|c|c|c|}
\hline Columna & Comunidad & Autor & Departamento \\
\hline 1 & Nolano spathulatae-Palauetum dissectae & Galán de Mera et al. (2009), Tab. 5 & Arequipa \\
\hline 2 & Hoffmannseggio mirandae-Palauetum weberbaueri & Galán de Mera et al. (2009), Tab. 5 & Arequipa \\
\hline 3 & Nolanetum scaposo-spathulatae & Este trabajo & Arequipa \\
\hline 4 & Palauetum camanensis-weberbaueri & Este trabajo & Arequipa \\
\hline 5 & Comunidad de Atriplex rotundifolia y Ephedra americana & Este trabajo & Arequipa \\
\hline 6 & Haageocereo olowinskiani-Anthericetum eccremorrhizi & Müller (1985), Tab. 3 & Lima \\
\hline 7 & Tetragonio crystallinae-Nolanetum humifusae & Müller (1985), Tab. 4 inventarios 1-4 & Lima \\
\hline 8 & Tetragonio-Nolanetum subasociación de Palaua rhombifolia & Müller (1985), Tab. 4 inventarios 5-6 & Lima \\
\hline 9 & Calandrinio paniculatae-Nolanetum humifusae & Müller (1985), Tab. 5 & Lima \\
\hline 10 & $\begin{array}{l}\text { Drymario weberbaueri-Nolanetum gayanae subasociación de } \\
\text { Oxalis bulbigera }\end{array}$ & Müller (1985), Tab. 6 inventarios 1-6 & Lima \\
\hline 11 & Drymario weberbaueri-Nolanetum gayanae & $\begin{array}{l}\text { Müller (1985), Tab. } 6 \text { inventarios } \\
\text { 7-10 }\end{array}$ & Lima \\
\hline 12 & $\begin{array}{l}\text { Palauo rhombifoliae-Nolanetum gayanae subasociación de } \\
\text { Nolana humifusa }\end{array}$ & Müller (1985), Tab. 7 inventarios 1-2 & Lima \\
\hline 13 & Palauo rhombifoliae-Nolanetum gayanae & Müller (1985), Tab. 7 inventarios 3-4 & Lima \\
\hline 14 & $\begin{array}{l}\text { Palauo rhombifoliae-Nolanetum gayanae subasociación de } \\
\text { Urocarpidium peruvianum }\end{array}$ & Müller (1985), Tab. 7 inventarios 5-9 & Lima \\
\hline 15 & $\begin{array}{l}\text { Hymenocallido amancaes-Trixetum paradoxae subasociación de } \\
\text { Eragrostis peruviana }\end{array}$ & Müller (1985), Tab. 8 inventarios 1-5 & Lima \\
\hline 16 & Hymenocallido amancaes-Trixetum paradoxae & $\begin{array}{l}\text { Müller (1985), Tab. } 8 \text { inventarios } \\
6-18\end{array}$ & Lima \\
\hline 17 & $\begin{array}{l}\text { Hymenocallido amancaes-Trixetum paradoxae subasociación de } \\
\text { Valeriana chaerophylloides }\end{array}$ & $\begin{array}{l}\text { Müller (1985), Tab. } 8 \text { inventarios } \\
\text { 19-20 }\end{array}$ & Lima \\
\hline 18 & $\begin{array}{l}\text { Paspalo penicillati-Valerianetum chaerophylloidis subasociación de } \\
\text { Geranium limae }\end{array}$ & Müller (1985), Tab. 9 inventarios 1-4 & Lima \\
\hline 19 & Paspalo penicillati-Valerianetum chaerophylloidis & $\begin{array}{l}\text { Müller (1985), Tab. } 9 \text { inventarios } \\
\text { 5-12 }\end{array}$ & Lima \\
\hline 20 & $\begin{array}{l}\text { Paspalo penicillati-Valerianetum chaerophylloidis subasociación de } \\
\text { Erodium moschatum }\end{array}$ & $\begin{array}{l}\text { Müller (1985), Tab. } 9 \text { inventarios } \\
\text { 13-17 }\end{array}$ & Lima \\
\hline 21 & $\begin{array}{l}\text { Loaso nitidae-Commelinetum fasciculatae subasociación de } \\
\text { Alstroemeria pelegrina }\end{array}$ & $\begin{array}{l}\text { Müller (1985), Tab. } 10 \text { inventarios } \\
1-4\end{array}$ & Lima \\
\hline 22 & Loaso nitidae-Commelinetum fasciculatae & $\begin{array}{l}\text { Müller (1985), Tab. } 10 \text { inventarios } \\
5-7\end{array}$ & Lima \\
\hline 23 & Loaso nitidae-Commelinetum fasciculatae subasociación de Trixis paradoxa & $\begin{array}{l}\text { Müller (1985), Tab. } 10 \text { inventarios } \\
8-9\end{array}$ & Lima \\
\hline 24 & Legousio biflorae-Centaurietum lomae & Müller (1985), Tab. 13 & Lima \\
\hline 25 & Nicotiano paniculatae-Urocarpidetum peruvianae & $\begin{array}{l}\text { Müller (1985), Tab. } 14 \text { inventarios } \\
1-3\end{array}$ & Lima \\
\hline 26 & $\begin{array}{l}\text { Nicotiano paniculatae-Urocarpidetum peruvianae subasociación de } \\
\text { Urtica urens }\end{array}$ & $\begin{array}{l}\text { Müller (1985), Tab. } 14 \text { inventarios } \\
4-6\end{array}$ & Lima \\
\hline 27 & Philoglosso peruvianae-Urocarpidetum peruviani & Galán de Mera et al. (2002), Tab. 5 & $\begin{array}{l}\text { Lima, Ica, } \\
\text { La Libertad }\end{array}$ \\
\hline
\end{tabular}




\section{CUADRO 3}

Inventarios levantados por los autores, comunidades vegetales y sus unidades superiores

TABLE 3

Releves make up by the authors, plant communities and higher units

Número de inventario

Altitud (m)

$\operatorname{Area}\left(\mathbf{m}^{2}\right)$

\section{Pendiente (\%)}

Características de asociaciones y alianzas

Nolana spathulata

Palaua velutina

Leptoglossis lomana

Spergularia congestifolia

Palaua weberbaueri

Nolana confinis

Tiquilia elongata

Tetragonia vestita

Nolana scaposa

Dictyophragmus englerianus

Weberbauerella brongniartioides

Lupinus mollendoensis

Hoffmannseggia miranda

Nolana pallidula

Cryptantha parviflora

Palaua camanensis

Oxalis sepalosa

Malesherbia angustisecta

Diferenciales de subasociación

Haageocereus decumbens

Forma con ganadería

Chenopodium petiolare

Loasa urens

Características de orden y clase

Palaua dissecta

Eragrostis peruviana

Plantago limensis

Monnina macrostachya

Argylia radiata

Oenothera arequipensis

Tetragonia crystallina

Nolana gayana

Cristaria multifida

Tiquilia litoralis

\begin{tabular}{|c|c|c|c|c|}
\hline & 11 & 22 & 2 & \\
\hline 45678 & 90123 & 4567890123 & 5 & \\
\hline & & & & \\
\hline 2222222 & 33333 & 3223325443 & 5 & \\
\hline 222022 & 33444 & 642153716 & 8 & 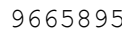 \\
\hline 5045 & & 165345733 & & 2 \\
\hline 1111 & 111 & $111 \quad 11 \quad 11$ & 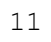 & \\
\hline 0000000 & 000 & 00500500 & 0 & 022 \\
\hline 000000 & 00 & 00000000 & 0 & \\
\hline 444 & 212 & 5544432 & 4 & \\
\hline 50 & & $5-5055500$ & 0 & - \\
\hline 222 & $1 \ldots$ & $+112+2332$ &.+ & \\
\hline $1+11111$ & $\ldots$ & $1+.+\ldots$ & • & \\
\hline $1 \ldots 11$ & $1 \ldots$ & $\ldots 1 \ldots 1$ & .1 & \\
\hline$\ldots \ldots$ & 122 & $\ldots 1.1 \ldots$ & - & \\
\hline$\ldots \ldots$ & 34444 & $\ldots \ldots \ldots$ & 3 & $\ldots$ \\
\hline$\cdots \cdots$ & $1 \ldots$ & $\ldots \ldots+.+$ & - & $.1+$ \\
\hline$\cdots \cdots$ & $\cdots$ & $211221 \ldots 2$ & & \\
\hline$\ldots \ldots$ & $\ldots$ & $1 \ldots$ & . & \\
\hline$\ldots \ldots$ & $\cdots$ & $2+.13 \ldots 11$ & $\cdots$ & \\
\hline$\ldots \ldots 11$ & $\ldots$ & $\ldots 1 \ldots+1$ & . & \\
\hline$\ldots 121.2$ & & & $\cdots$ & \\
\hline$+.2+\ldots+$. & $\cdots$ & $\ldots$ & $\cdots$ & \\
\hline$\ldots \ldots$ & $1+\ldots 1$ & . & 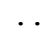 & \\
\hline$+.2 \ldots+\ldots$ & $\cdots$ & $\cdots$ & $\cdots$ & \\
\hline$\ldots \ldots$ & $\ldots$ & $1.2 \ldots \ldots$ & . & \\
\hline$\cdots$ & & & 11 & \\
\hline$\ldots$ & . & & . & \\
\hline$\cdots \cdots$ & . & & & \\
\hline$\cdot$ & & $.11+1$ & & \\
\hline . &.+.1 & 43 & 24 & \\
\hline$\cdots$ & $\ldots 1$ & $.1 .+$. & + . & \\
\hline 11233332 & $.1+\ldots$ & 2332131111 & . & \\
\hline$\ldots \ldots 1.1$ & 11111 & ...111. & 1. & \\
\hline $11 \ldots 11$ & $++\ldots$ & $\ldots \ldots$ & 1. & \\
\hline $11 \ldots+11$ & $\ldots$ & & . & \\
\hline$\cdots \ldots$ & & $\ldots 1 \ldots$ & . & \\
\hline$\ldots \ldots 1$ & $\ldots++$ & & 1. & \\
\hline$\ldots .$. & & $\ldots \ldots \ldots+$ & ++ & \\
\hline$\ldots$ & $\cdots$ & $\ldots 2 \ldots 1 \ldots$ & $\cdots$ & \\
\hline . & $\cdots$ & $\cdots \cdots$ & $\cdots$ & $\cdot+.+$ \\
\hline & & & & \\
\hline
\end{tabular}


CUADRO 3 (COntinuación)

Inventarios levantados por los autores, comunidades vegetales y sus unidades superiores

TABLE 3 (Continued)

Releves make up by the authors, plant communities and higher units

Número de inventario
Verbena pogostoma
Gilia glutinosa
Loasa fruticosa
Cistanthe celosioides
Tiquilia dichotoma
Tiquilia elongata
Com. Atriplex rotundifolia-Ephedra americana
Atriplex rotundifolia
Schinus molle
Lycopersicon pennellii
Ephedra americana
Ophryosporus hoppii
Ambrosia artemisioides
Tessaria absinthioides
Alternanthera arequipensis
Tarasa operculata
Compañeras
Eragrostis nigricans
Hoffmannseggia prostrata
Onoseris odorata
Talinum paniculatum
Cenchrus brownii
Galinsoga sp.
Grindelia glutinosa
Encelia canescens
Cistanthe weberbaueri
Sonchus oleraceus
Nothoscordum bivalve

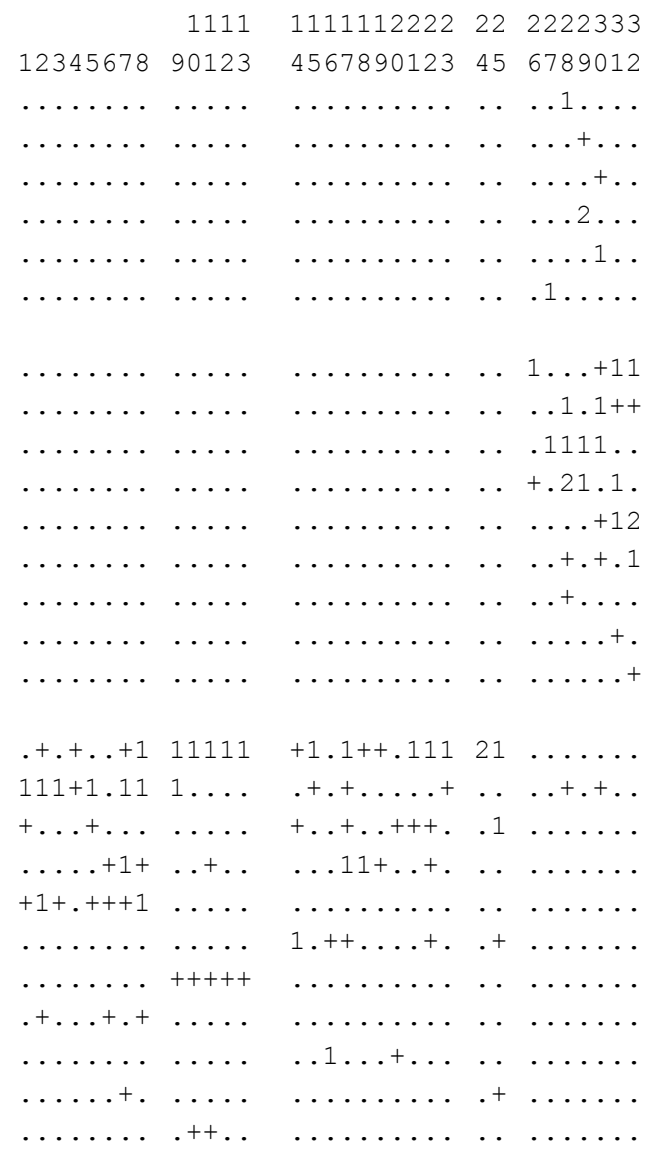

Compañeras con una presencia: Gamochaeta purpurea 12:+; Heliotropium krauseanum 17:1; Vulpia megalura 30:1; Portulaca perennis 1:+; Chamaesyce serpens 29:+; Linaria canadensis 12:+.

Localidades: 1-13- Arequipa, lomas de Mejía [1- 19K 0192035, 8107927; 2- 19K 0192245, 8107813; 3- 19K 0192295, 8107815; 4- 19K 0191843, 8108197; 5- 19K 0191704, 8108327; 6- 19K 0191893, 8107849; 7- 19K 0191903, 8107853; 8- 19K 0192315, 8107789; 9- 19K 0192335, 8108129; 10- 19K 0192337, 8108168; 11- 19K 0192360, 8108179; 12- 19K 0192407, 8108177; 13- 19K 0192232, 8108304]; 14-25- Arequipa, lomas de Camaná [14- 18K 0746617, 8165622; 1518K 0746236, 8165440; 16- 18K 0745983, 8165369; 17- 18K 0746678, 8165781; 18- 18K 0746659, 8165716; 19- 18K 0746163, 8165411; 20- 18K 0747541, 8166757; 21- 18K 0747412, 8166638; 22- 18K 0747244, 8166328; 23- 18K 0747049, 8165992; 24- 18K 0747658, 8166716; 25- 18K 0747650, 8166797]; 26- Arequipa, de Ocoña a Camaná [18K 0713167, 8176677]; 27-32- Arequipa, de Atico a Caravelí [27- 18K 0649270, 8225936; 28- 18L 0660523, 8243601; 29- 18L 0660523, 8243601; 30- 18L 0661695, 8243997; 31- 18L 0669291, 8245328; 32- 18L 0668961, 8245949].

Correspondencia de la escala de Braun-Blanquet: +: $<1 \%, 1: 1-5 \%, 2:$ 5-25\%, 3: 25-50\%, 4: 50-75\%, 5: $>75 \%$. 

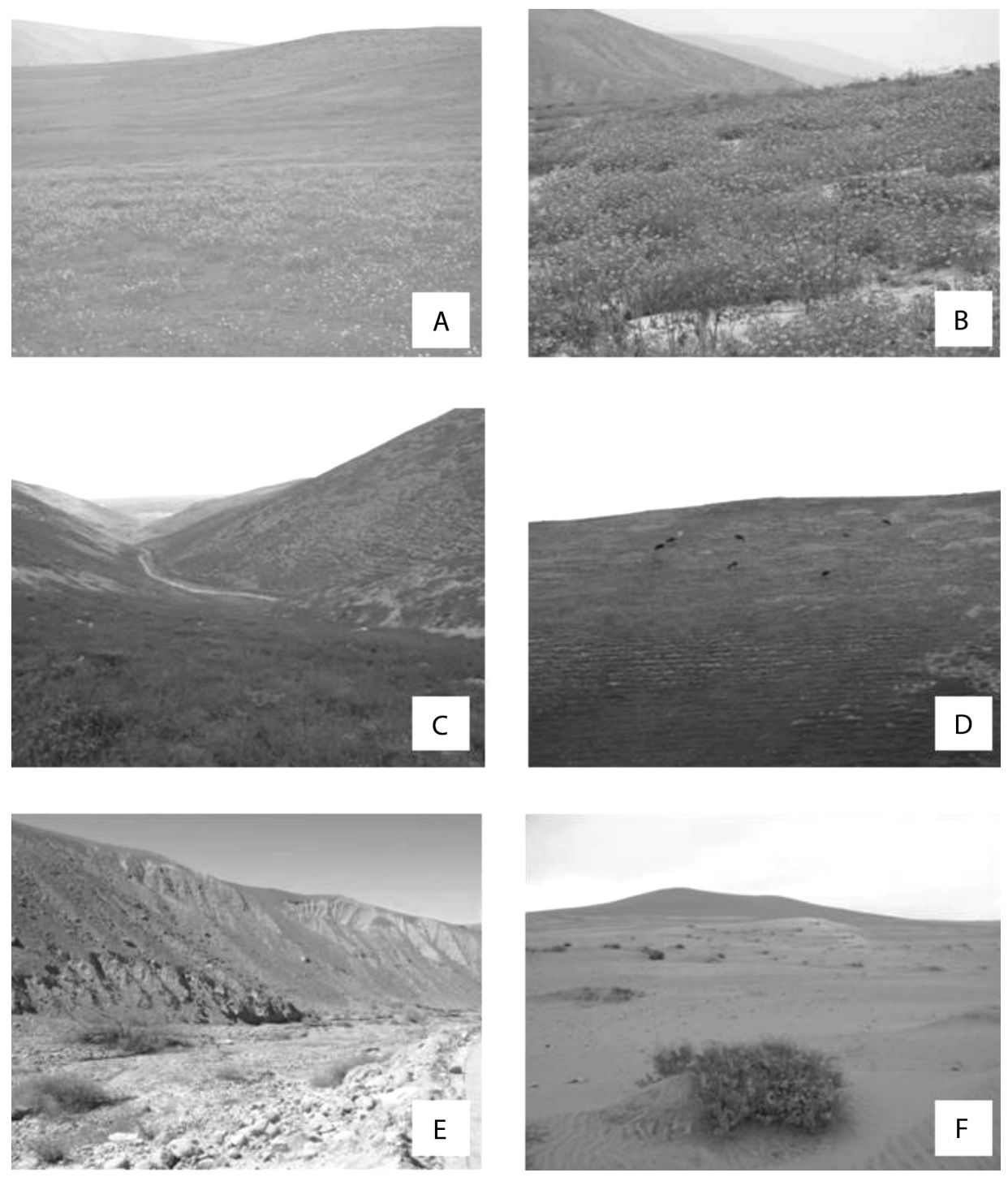

Fig. 1. Comunidades vegetales. (A) Nolano spathulatae-Palauetum dissectae y (B) Hoffmannseggio mirandae-Palauetum weberbaueri, en las lomas de Mejía; (C) Nolanetum scaposo-spathulatae y (D) Palauetum camanensis-weberbaueri, en las lomas de Camaná; (E) Comunidad de Atriplex rotundifolia y Ephedra americana con Schinus molle en el valle AticoCaravelí; (F) Comunidad marginal de Atriplex rotundifolia y Ephedra americana entre Ocoña y Camaná.

Fig. 1. Plant communities. (A) Nolano spathulatae-Palauetum dissectae and (B) Hoffmannseggio mirandae-Palauetum weberbaueri, in the lomas of Mejía; (C) Nolanetum scaposo-spathulatae and (D) Palauetum camanensis-weberbaueri, in the lomas of Camaná; (E) Atriplex rotundifolia and Ephedra americana plant community with Schinus molle in the Atico-Caravelí valley; (F) Atriplex rotundifolia and Ephedra americana marginal community between Ocoña and Camaná. 
Hoffmannseggia miranda, Palaua weberbaueri y Spergularia congestifolia.

\subsubsection{Nolanetum scaposo-spathulatae} ass. nova (Cuadro 1, columna 3; Cuadro 3, inventarios 14-23; holotypus: Cuadro 3, inventario 14): Esta vegetación efímera de las lomas de Camaná, tapiza los suelos arenosos poco estabilizados a una altitud que oscila entre 250 y 500m (Fig. 1C). En su combinación florística participa Dictyophragmus englerianus, Leptoglossis lomana, Nolana scaposa, N. spathulata, Palaua velutina y Tetragonia vestita.

Las áreas con pendientes pedregosas se enriquecen con Haageocereus decumbens (Vaupel) Backeb., y se puede describir la subasociación haageocereetosum decumbentis subass. nova (holotypus: Cuadro 3, inventario 23).

\subsubsection{Palauetum camanensis-weberbaue-} ri ass. nova (Cuadro 1, columna 4; Cuadro 3, inventarios 24-25; holotypus: Cuadro 3, inventario 24): Ésta es la asociación de los suelos arenosos estabilizados de las lomas de Camaná, cuya altitud alcanza más de $600 \mathrm{~m}$. Los elementos de su combinación florística característica son: Leptoglossis lomana, Nolana spathulata, Palaua camanensis, P. weberbaueri y Tetragonia crystallina L'Hér.

El uso de estas dos asociaciones para el pastoreo con ganado vacuno (Fig. 1D) conlleva a la presencia constante de Chenopodium petiolare Kunth y Loasa urens, por lo que podemos determinar una forma de ambas asociaciones que comienza a enriquecerse con plantas nitrófilas, y se marca así una transición con la alianza nitrófila Loasion urentis (Galán de Mera et al. 2002).

\subsection{Tetragonio crystallinae-Plantagine-} talia limensis ordo novo hoc loco (holotypus: Nolanion spathulatae all. nova): Las dos alianzas reconocidas en el Perú pueden reunirse en este nuevo orden que, mediante sus características, distinguen a las lomas peruanas de las que se encuentran en Chile entre Copiapó y la Quebrada de Camarones (Arica), espacio donde la combinación de especies cambia totalmente
(Rundel et al. 1991, Galán de Mera et al. 1997, Pinto \& Luebert 2009)(por ejemplo, Ephedra breana Phil., Fagonia chilensis Hook. \& Arn., Malesherbia tocopillana Ricardi, Nolana divaricata I.M. Johnst., N. mollis I.M. Johnst., $N$. sedifolia Poepp., N. tocopillensis I.M. Johnst., Oxalis gigantea Barnéoud, Tetragonia angustifolia Barnéoud, etc.), y constituyen en el extremo $\mathrm{N}$ de Chile una barrera biogeográfica que separa dos grandes unidades de vegetación de lomas (Dillon et al. 2003).

Entonces, son especies características de este nuevo orden: Calandrinia alba (Ruiz \& Pav.) DC., Cryptantha limensis I.M. Johnst., Dyschoriste repens (Nees) Kuntze, Eragrostis peruviana (Jacq.) Trin., Linum parvum I.M. Johnst., Monnina macrostachya Ruiz \& Pav., Oxalis lomana Diels, O. solarensis R. Knuth, Palaua malvifolia Cav., Pectocarya lateriflora (Lam.) DC., Plantago limensis Pers., Spananthe paniculata var. peruviana $\mathrm{H}$. Wolff, Tetragonia crystallina y Vicia lomensis J.F. Macbr.

1.4. La vegetación de las quebradas. Comunidad de Atriplex rotundifolia y Ephedra americana (Cuadro 1, columna 5; Cuadro 3 , inventarios 26-32): Las quebradas por donde discurre el agua en la época de nieblas o en los años de influencia de El Niño, se enriquecen sobre todo con Atriplex rotundifolia Dombey ex Moq., que además se asienta en áreas donde se perciben eflorescencias salinas. A. rotundifolia en las quebradas más próximas a los Andes, en la barrera interdesértica, se acompaña de Schinus molle L., Pluchea absinthioides (Hook. \& Arn.) H. Rob., Ophryosporus hoppii (B.L. Rob.) R.M. King \& H. Rob. o Alternanthera arequipensis Suess. (Fig. 1E), plantas que forman parte de la alianza Plucheion absinthioidis (Galán de Mera et al. 2009), y a su vez, de elementos de las lomas que han encontrado una mayor humedad en este medio (Gilia glutinosa Phil., Loasa fruticosa (Phil.) Urb. \& Gilg, Malesherbia angustisecta Harms, Tiquilia litoralis (Phil.) A.T. Richardson, Verbena pogostoma Klotzsch, etc.). La vegetación con $A$. rotundifolia, que es leñosa, a medida que nos desplazamos hacia la 
costa se va empobreciendo al ser ahogada por la arena de las dunas (Fig. 1F), y forma una comunidad marginal de Atriplex y Ephedra americana en el sentido de Foucault (1981). Esta situación de empobrecimiento se puede observar con claridad en el valle de CaravelíAtico (18K 0649270, 8225936) y en las dunas interiores situadas entre Ocoña y Camaná (18K 0713167, 8176677).
2. Diversidad: Como consecuencia de aplicar el índice de diversidad de ShannonWiener sobre las columnas del Cuadro 1 , y de enfrentarlo al número de especies ruderales o nitrófilas, hemos obtenido la gráfica de la (Fig. 2). En ella podemos observar cómo un aumento de especies ruderales, favorecidas por la actividad ganadera intensiva, generalmente conlleva a un aumento del valor del índice de diversidad.

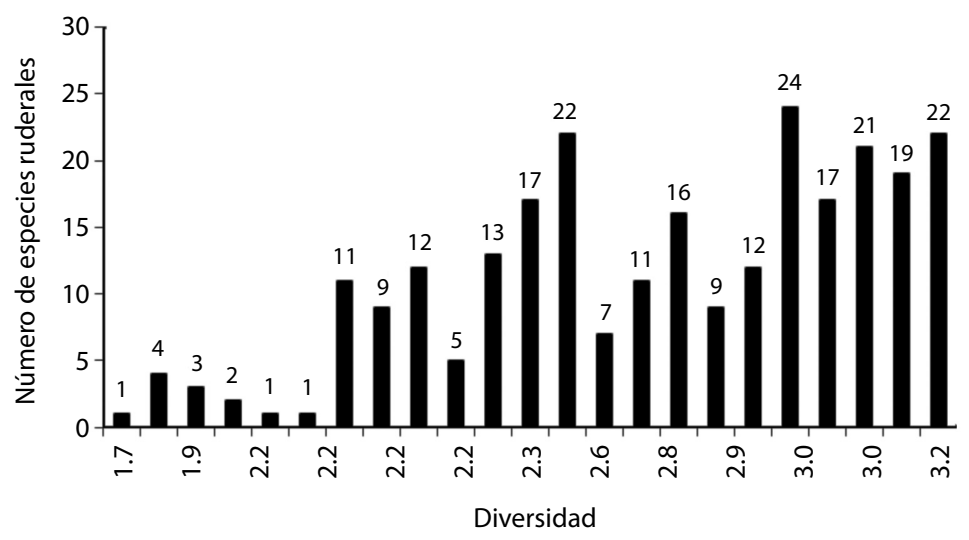

Fig. 2. Variación de la diversidad frente al número de plantas ruderales de las columnas del cuadro 1.

Fig. 2. Variation of diversity vs. number of ruderal plants from the columns of the table 1 .

\section{DISCUSIÓN}

1. Aspectos sintaxonómicos: En un trabajo anterior (Galán de Mera et al. 2002) describimos el orden de vegetación nitrófila andina y pacífica Commelinetalia fasciculatae con la alianza Loasion urentis y la asociación Philoglosso peruvianae-Urocarpidetum peruviani (Cuadro 1, columna 27), en cuya combinación florística podemos encontrar Chenopodium petiolare, Commelina fasciculata Ruiz \& Pav., Loasa urens, Parietalia debilis G. Forst., Philoglossa peruviana DC. y Urocarpidium peruvianum (L.) Krapov. Esta vegetación está muy extendida en las lomas debido a la influencia de la ganadería, y dicho orden, constituye el tipo de la clase Palauo dissectae-Nolanetea gayanae (Galán de Mera 2005).
El orden Tetragonio crystallinae-Plantaginetalia limensis representa a las alianzas y asociaciones que no están ligadas a la nitrificación del suelo, aunque a veces pueden enriquecerse con plantas nitrófilas, como Chenopodium petiolare o Loasa urens por acción incipiente de la ganadería.

Muy diferente en su composición florística es la vegetación de las quebradas, cuyos elementos se hacen más escasos al acercarnos a la costa. La asociación Plucheetum absinthioidis (Galán de Mera et al. 2009) se sitúa en áreas areno-limosas inundadas permanentemente por las aguas salobres de los caños y acequias derivadas de los ríos andinos. Desde el punto de vista sintaxonómico, pertenece a la alianza Plucheion absinthioidis que llega 
muy empobrecida a las quebradas muy áridas de la barrera interdesértica y a las dunas de la costa, con apenas algunas plantas (Cuadro 3, inventarios 26-32). Si en el desierto peruano las plantas principales son Atriplex rotundifolia y Ephedra americana acompañadas, sobre todo, de Pluchea absinthioides y Schinus molle, en el de Atacama (Chile), las características son Acantholippia trifida (Gay) Moldenke, Atriplex atacamensis Phil., Distichlis spicata (L.) Greene y Pluchea absinthioides (Rundel et al. 1991).

En síntesis, el esquema sintaxonómico de las unidades de vegetación tratadas en este trabajo es el siguiente:

I. Palauo dissectae-Nolanetea gayanae Galán de Mera 2005

+ Commelinetalia fasciculatae Galán de Mera \& Rosa in Galán de Mera, Rosa \& Cáceres 2002

- Loasion urentis Galán de Mera \& Rosa in Galán de Mera, Rosa \& Cáceres 2002

1. Philoglosso peruvianae-Urocarpidetum peruviani Galán de Mera \& Rosa in Galán de Mera, Rosa \& Cáceres 2002

+ Tetragonio crystallinae-Plantaginetalia limensis ordo novo

- Nolanion humifusae all. nova

2. Tetragonio crystallinae-Nolanetum gayanae Müller 1985

3. Palauo rhombifoliae-Nolanetum gayanae Müller 1985

- Nolanion spathulatae all.nova

4. Nolano spathulatae-Palauetum dissectae Galán de Mera, Linares, Campos \& Vicente Orellana 2009

5. Hoffmannseggio mirandae-Palauetum weberbaueri Galán de Mera, Linares, Campos \& Vicente Orellana 2009

6. Nolanetum scaposo-spathulatae ass. nova 6a. haageocereetosum decumbentis subass. nova

7. Palauetum camanensis-weberbaueri ass. nova

II. Tessario integrifoliae-Baccharidetea salicifoliae Rivas-Martínez \& Navarro in Navarro \& Maldonado 2002
+ Plucheo absinthioidis-Baccharidetalia salicifoliae Rivas-Martínez \& Navarro in Navarro \& Maldonado 2002

- Plucheion absinthioidis Galán de Mera, Linares, Campos \& Vicente Orellana 2009

8. Plucheetum absinthioidis Galán de Mera, Linares, Campos \& Vicente Orellana 2009

9. Comunidad de Atriplex rotundifolia y Ephedra americana

\section{La vegetación de las lomas y los apor- tes de la historia}

2.1. Diversidad y composición florística:

Del Cuadro 1 (columnas 6-26) se desprende que algunas de las asociaciones creadas por Müller (1985) están muy enriquecidas por plantas ruderales debidas a la acción de la ganadería, de tal forma que llegan a constituir comunidades derivadas (Schaminée \& Stortelder 1996), donde dichas plantas ruderales han ido ganando terreno en el seno de las asociaciones centroperuanas del Tetragonio crystallinae-Nolanetum gayanae -de suelos arenosos poco estabilizados- y del Palauo rhombifoliaeNolanetum gayanae, de suelos arenosos consolidados. Los datos de las floras europea y norteamericana (Tutin et al. 1964-1980, Flora of North America Committee 1992-2007, Solomon 2010) nos han permitido diferenciar en el Cuadro 1 entre especies ruderales autóctonas -Cyclospermum laciniatum (DC.) Constance, Nicotiana paniculata L., Parietaria debilis G. Forst. o Urocarpidium peruvianum- y especies ruderales introducidas -Erodium cicutarium (L.) L'Hér. ex Aiton, E. moschatum (L.) L'Hér. ex Aiton, Sonchus oleraceus L. o Torilis nodo$s a$ (L.) Gaertn.- que llegan a invadir las áreas propias de las especies no nitrófilas, como Cryptantha granulosa I.M. Johnst., Nolana gayana (Gaudich.) Koch y Oenothera arequipensis Munz \& I.M. Johnst. Este aspecto invasor de la vegetación de las lomas por plantas nitrófilas ya fue advertido por Müller (1988) tras crear un número de asociaciones demasiado elevado en las lomas del centro del Perú, donde intervienen incluso elementos fisurícolas (León et al. 2002). En las lomas del sur las plantas ruderales son muy escasas, aunque 
podemos destacar una forma fitosociológica con Chenopodium petiolare y Loasa urens debido al uso intensivo de la ganadería vacuna en algunas localidades (Cuadro 3).

El índice de Shannon-Wiener (Fig. 2) nos permite comprobar que el aumento de la diversidad es debido a la introducción de plantas ruderales, precisamente en los inventarios del centro del Perú, donde las lomas se encuentran insuficientemente conservadas a pesar de formar parte de áreas reservadas (Brack Egg \& Mendiola Vargas (2004); sin embargo, las lomas del sur, más ricas en endemismos (Galán de Mera et al. 1997), no presentan ninguna fórmula de protección.

\subsection{Vegetación y uso del territorio:}

Existen datos del uso antrópico de distintas áreas del Desierto Pacífico desde tiempos prehistóricos, tanto en Perú (Beresford-Jones et al. 2009) como en Chile (Gallardo 2009). Sobre todo este último autor, indica en el arte rupestre del desierto de Atacama la presencia de dibujos de camélidos; por tanto, es lógico pensar que la vegetación y la diversidad de la flora actual de las lomas son debidas a la ganadería. La cultura Inca y las etnias preincaicas -Chupaychu en el centro y Lupaqa en el sur del Perú- manejaban los cultivos y la ganadería a través del control vertical de un máximo de pisos ecológicos (Murra 2009), donde tenía una gran importancia el transporte de productos y la trashumancia entre la costa y los Andes (Bennett \& Bird 1949, Díez de San Miguel 1964); la vegetación de las lomas era esencial para el sustento de las llamas que eran transportadas con fines rituales, para carga de productos serranos o costeños, o para aprovechar los pastos suculentos de las lomas, mientras en los Andes estaban secos. Incluso podemos encontrar ruinas de los asentamientos preincaicos que servían para gestionar los pastos y cultivos de las lomas. Hoy en día sigue existiendo trashumancia entre los Andes y la costa, lo que hace pensar en la dispersión zoócora de grupos de plantas altoandinas hacia la costa como indican algunos autores (Tapia Núñez \& Flores Ochoa 1984). En el Cuadro 1 podemos observar que las plantas ruderales autóctonas, como Parietaria debilis, Cyclospermum laciniatum, Urocarpidium peruvianum, Chenopodium petiolare, Solanum montanum L. y Nicotiana paniculata, también se encuentran en los pisos ecológicos medios de los Andes (Brako \& Zarucchi 1993) formando parte de comunidades que se encuentran en torno a las poblaciones (Galán de Mera et al. 2004).

Como señalan Armesto \& Vidiella (1993), la entrada de plantas europeas en el Desierto Pacífico de Chile se realizó en tiempos recientes. En el Perú, la invasión española fue el origen de la presencia de plantas europeas, como Sonchus oleraceus, Erodium moschatum, E. malacoides (L.) L'Hér. ex Aiton y Medicago polymorpha L. (Cuadro 1), por introducción de su propia ganadería y de especies exóticas. El caso de M. polymorpha es muy característico, ya que es una planta forrajera ampliamente cultivada en el piso supratropical andino (Ruiz Canales \& Tapia Núñez 1987), y cuyos frutos hacen uso de la exozoocoria como forma de dispersión. Muchas de estas especies se naturalizaron tras la fractura del modelo agropastoril incaico al crear las haciendas de la costa (Murra 2009), y dependen del sistema ganadero actual.

\section{AGRADECIMIENTOS}

Deseamos expresar nuestro agradecimiento a María Angélica Matarazzo de Benavides por su información sobre la trashumancia en el Perú, y a Andrea Galán Salazar por la revisión de los párrafos en inglés. Este trabajo ha sido realizado con la ayuda de la Cátedra de Desarrollo Sostenible y Medio Ambiente de la Universidad San Pablo-CEU y la Fundación Banco de Santander, en el marco de los convenios firmados entre la Universidad San Pablo-CEU, la Universidad Nacional Mayor de San Marcos en Lima (Perú) y la Universidad Nacional de San Agustín en Arequipa (Perú).

\section{RESUMEN}

La vegetación de las lomas, causada sobre todo por las precipitaciones debidas a la influencia de la corriente 
fría de Humboldt, constituye la mayor originalidad del desierto peruano y chileno, con un alto número de endemismos que forman asociaciones geovicarias. En este trabajo, presentamos un estudio fitosociológico sobre la vegetación de los medios arenosos de las lomas del desierto costero peruano. De acuerdo con el método de Braun-Blanquet, hemos levantado 32 inventarios fitosociológicos a los que hemos añadido 138 procedentes de la bibliografía. En cada inventario, hemos analizado la composición florística y distintos parámetros ecológicos, como la altitud, el suelo y la geomorfología. Todos los inventarios fueron sintetizados en una tabla para deducir las asociaciones, las unidades fitosociológicas superiores y el origen de su flora. Como resultado, describimos dos nuevas asociaciones del sur del Perú -Nolanetum scaposo-spathulatae y Palauetum camanensis-weberbaueri, dos alianzas -Nolanion humifusae, del centro del Perú, y Nolanion spathulatae del sur del Perú-, y un nuevo orden -Tetragonio crystallinae-Plantaginetalia limensis-. También se presenta la vegetación de las quebradas en el contexto de las comunidades de riberas de la costa y su alteración por las dunas. Después de la aplicación del índice de Shannon-Wiener sobre las columnas de la tabla sintética, podemos deducir que el aumento de especies ruderales andinas y europeas en la costa central del Perú se ha visto favorecido por una actividad ganadera intensiva. La trashumancia entre los Andes y la costa, desde tiempos preincaicos hasta la actualidad, ha producido la dispersión zoócora de plantas altoandinas hacia la costa; la presencia de plantas europeas tuvo su origen en la colonización española de la costa del Perú.

Palabras clave: Perú, desierto, fitosociología, diversidad, culturas pre-Inca/Inca, invasión española, uso del territorio.

\section{REFERENCIAS}

Ackermann, W. \& W. Durca. 1997. SORT 4.1. Processing of vegetation releves and species lists. Edición del autor, Leipzig, Alemania.

Armesto, J.J. \& P.E. Vidiella. 1993. Plant life-forms and biogeographic relations of the flora of Lagunillas $\left(30^{\circ} \mathrm{S}\right)$ in the fog-free Pacific coastal desert. Ann. Mo. Bot. Gard. 80: 499-511.

Arntz, W.E. \& E. Fahrbach. 1996. El Niño. Experimento climático de la naturaleza. Fondo de Cultura Económica, México D.F., México.

Bennett, W.C. \& J.B. Bird. 1949. Andean Culture History. American Museum of Natural History, Nueva York, Nueva York, EEUU.

Beresford-Jones, D., H. Lewis \& S. Boreham. 2009. Linking cultural and environmental change in Peruvian prehistory: Geomorphological survey of the Samaca Basin, Lower Ica Valley, Peru. Catena 78: 234-249.
Brack Egg, A. \& C. Mendiola Vargas. 2004. Ecología del Perú. Bruño, Lima, Perú.

Brako, L. \& J.L. Zarucchi. 1993. Catálogo de las Angiospermas y Gimnospermas del Perú. Missouri Botanical Garden, San Luis, Misuri, EEUU.

Braun-Blanquet, J. 1932. Plant Sociology. The study of plant communities. Koeltz, Koenigstein, Alemania.

Bruns, F. 1929. Beiträge zur Kenntnis der Vegetation des peruanischen Küstengebietes. Universidad de Hamburgo, Hamburgo, Alemania.

Cieza de León, P. 1988 [1553]. La crónica del Perú. Peisa, Lima, Perú.

Deil, U. 2003. Characters of "traditional" and "modern" vegetation landscapes- a comparison of Northern Morocco and Southern Spain. Phytocoenologia 33: 819-860.

Dierschke, H. 1994. Grundlagen und Methoden der Pflanzensoziologie. Ulmer, Sttutgart, Alemania.

Díez de San Miguel, G. 1964[1567]. Visita hecha a la provincia de Chucuito. Casa de la Cultura, Lima, Perú.

Dillon, M.O., M. Nakazawa \& S. Leiva. 2003. The Lomas Formations of Coastal Peru: Composition and Biogeographic History, p. 1-9. In J. Haas \& M.O. Dillon (eds.). El Niño in Peru: Biology and Culture Over 10000 Years. Field Museum of Natural History, Chicago, Illinois, EEUU.

Dillon, M.O., T. Tu, A. Soejima, T. Yi, Z. Nie, A. Tye \& J. Wen. 2007. Phylogeny of Nolana (Nolaneae, Solanoideae, Solanaceae) as inferred from granulebound starch synthase I (GBSSI) sequences. Taxon 56: $1000-1011$.

Ellenberg, H. 1959. Über den Wasserhaushalt tropischer Nebeloasen in der Küstenwüste Perus. Ber. Geobot. Forschungsinst. Rübel Zürich 1958: 47-74.

Ferreyra, R. 1953. Comunidades vegetales de algunas lomas costaneras del Perú. Bol. Estac. Exp. Agríc. "La Molina" 53: 1-88.

Ferreyra, R. 1960. Algunos aspectos fitogeográficos del Perú. Publ. Institut. Geogr. UNMSM, serie I (B): 41-87.

Ferreyra, R. 1983. Los tipos de vegetación de la costa peruana. Anales Jard. Bot. Madrid 40: 241-256.

Ferreyra, R. 1993. Registros de la vegetación en la costa peruana en relación con el fenómeno El Niño. Bull. Institut. Franç. Études Andines 22: 259-266. 
Flora of North America Committee. 1992-2007. Flora of North America. Missouri Botanical Garden, San Luis, Misuri, EEUU.

Foucault, B. 1981. Réflexions sur 1'appauvrissement des syntaxons aux limites chorologiques des unités phytosociologiques supérieurs et quelques-unes de leurs consequences. Lazaroa 3: 75-100.

Galán de Mera, A., J.A. Vicente Orellana, J.A. Lucas García \& A. Probanza Lobo. 1997. Phytogeographical sectoring of the Peruvian coast. Global Ecol. Biogeogr. Lett. 6: 349-367.

Galán de Mera, A., R. Morales Alonso \& J.A. Vicente Orellana. 2000. Pasture communities linked to ovine stock. A synthesis of the Poetea bulbosae class in the Western Mediterranean Region. Phytocoenologia 30: 223-267.

Galán de Mera, A., M.V. Rosa \& C. Cáceres. 2002. Una aproximación sintaxonómica sobre la vegetación del Perú. Clases, órdenes y alianzas. Acta Bot. Malacitana 27: 75-103.

Galán de Mera, A., S. Baldeón, H. Beltrán, M. Benavente \& J. Gómez. 2004. Datos sobre la vegetación del centro del Perú. Acta Bot. Malacitana 29: 89-115.

Galán de Mera, A. 2005. Clasificación fitosociológica de la vegetación de la región del Caribe y América del Sur. Arnaldoa 12: 86-111.

Galán de Mera, A., E. Linares Perea, J. Campos de la Cruz \& J.A. Vicente Orellana. 2009. Nuevas observaciones sobre la vegetación del sur del Perú. Del Desierto Pacífico al Altiplano. Acta Bot. Malacitana 34: 107-144.

Gallardo, F. 2009. Social interaction and rock art styles in the Atacama Desert (northern Chile). Interaction sociale et styles d'art rupestre dans le désert de l'Atacama (nord du Chili). Antiquity 83: 619-633.

Huber, O. \& R. Riina. 1997. Glosario Fitoecológico de las Américas. Vol. I. América del Sur: Países hispanoparlantes. UNESCO-Fundación Instituto Botánico de Venezuela, Caracas, Venezuela.

Hueck, K. \& P. Seibert. 1981. Vegetationskarte von Südamerika. Fischer, Stuttgart, Alemania, Nueva York, Nueva York, EEUU.

IGM (Instituto de Geología y Minería). 1975. Mapa geológico del Perú. Lima, Perú.

Izco, J. \& M. del Arco. 2003. Código Internacional de Nomenclatura Fitosociológica. Servicio de
Publicaciones Universidad de La Laguna, Santa Cruz de Tenerife, España.

Jones, M. 1988. Archeology and the Flora of the British Isles. Human influence on the evolution of plant communities. Bot. Soc. Brit. Isles, Conf. Report 19: $39-52$.

Kopecký, K., J. Dostalek \& T. Frantik. 1995. The use of the deductive method of syntaxonomic classification in the system of vegetational units of the BraunBlanquet approach. Vegetatio 117: 95-112.

León, B., A. Cano \& K.R. Young. 2002. Los helechos de las lomas costeras del Perú. Arnaldoa 9: 7-42.

Magurran, A. 1989. Diversidad Ecológica y su Medición. Vedra, Barcelona, España.

Müller, G.K. \& C. Müller. 1974. Aus dem wissenschaftlichen Leben der Karl-Marx-Universität. Exkursionsberichte. Vegetationskundliche Studien in Peru (Teil II). Wiss. Z. Karl-Marx-Univ. Leipzig, Math.Naturwiss. R. 23: 683-699.

Müller, G.K. 1985. Zur floristichen Analyse der peruanischen Loma-Vegetation. Flora 176: 153-165.

Müller, G.K. 1988. Anthropogene Veränderungen der Loma-Vegetation Perus. Flora 180: 37-40.

Murra, J.V. 2009. El mundo andino. Población, medio ambiente y economía. Instituto de Estudios Peruanos/ Pontificia Universidad Católica del Perú, Lima, Perú.

Phillips, O. \& J.S. Miller. 2002. Global Patterns of Patterns of Plant Diversity: Alwyn H. Gentry's Forest Transect Data Set. Missouri Botanical Garden, San Luis, Misuri, EEUU.

Pinto, R. \& F. Luebert. 2009. Datos sobre la flora vascular del desierto costero de Arica y Tarapacá, Chile, y sus relaciones fitogeográficas con el sur del Perú. Gayana Botánica 66: 28-49.

Puig, H., A. Fabre, M.F. Bellan, D. Lacaze, F. Villasante \& A. Ortega. 2002. Déserts et richesse floristique: les lomas du sud péruvien, un potentiel à conserver. Sécheresse 13: 215-225.

Rivas-Martínez, S., D. Sánchez-Mata \& M. Costa. 1999. North American Boreal and Western temperate Forest Vegetation (Syntaxonomical sinopsis of the potential natural plant communities of North America, II). Itinera Geobot. 12: 5-316.

Ruiz Canales, C. \& M.E. Tapia Núñez. 1987. Producción y manejo de forrajes en los Andes del Perú. Pisa, Lima, Perú. 
Rundel, P.W., M.O. Dillon, B. Palma, H.A. Mooney, S.L. Gulmon \& J.R. Ehleringer. 1991. The phytogeography and ecology of the coastal Atacama and Peruvian deserts. Aliso 13: 1-49.

Schaminée, J.H.J. \& A.H.F. Stortelder. 1996. Recent developments in phytosociology. Acta Bot. Neerl. 45: 443-459.

Schwabe, A. 1997. Sigmachorology as a subject of phytosociological research: a review. Phytocoenologia 27: 463-507.

Sluyter, A. 2001. Colonialism and landscape in the Americas: Material/conceptual transformations and continuing consequences. Ann. Assoc. Am. Geogr. 91: 410-428.

Solomon, J. 2010. Tropicos. org. Missouri Botanical Garden, San Luis, Misuri, EEUU (Consultado: 7 mayo 2010, www.tropicos.org/).

Tapia Núñez, M.E. \& J.A. Flores Ochoa. 1984. Pastoreo y pastizales de los Andes del Sur del Perú. Instituto Nacional de Investigación y Promoción Agropecuaria, Lima, Perú.
Tutin, T.G., V.H. Heywood, N.A. Burges, D.H. Valentine, S.M. Walters \& D.A. Webb (eds.). 1964-1980. Flora Europea Cambrige University, Londres, Gran Bretaña.

Weberbauer, A. 1912. Pflanzengeographische Studien in südlichen Peru. Bot. Jahrb. Syst. 107: 27-46.

Weberbauer, A. 1936. Phytogeography of the Peruvian Andes, p. 13-81. In J.F. MacBride (ed.). Flora of Peru, Vol. 13/1. Field Museum of Natural History, Chicago, Illinois. EEUU.

Weberbauer, A. 1945. El mundo vegetal de los Andes Peruanos (Estudio fitogeográfico). Ministerio de Agricultura, Lima, Perú.

Wilmanns, O. 1997. Zur Geschichte der mitteleuropäischen Trockenrasen seit dem Spätglazial- Methoden, Tatsachen, Hypotesen. Phytocoenologia 27: 213-233.

Young, K.R. 2009. Andean land use and biodiversity: Humanized landscapes in a time of change. Ann. Mo. Bot. Gard. 96: 492-507. 